\title{
The effect of layers and bullet type on impact properties of Glass Fibre Reinforced polymer (GFRp) using a Single Stage Gas Gun (SSGG)
}

\begin{abstract}
The purpose of this work is to study the best number of layer with the higher impact energy using Glass Fibre Reinforced Polymer (GFRP). The number of layers used in this study was 25, 33, 41, and 49. The impact test was performed using Single Stage Gas Gun (SSGG) for each layers given above with different bullets such as blunt, hemispherical and conical bullets. The gas gun pressure was set to 5, 10, 15 and 20 bar. All of the signals captured from the impact test were recorded using a ballistic data acquisition system. The correlation between the impact energy in terms of number of layer and type of bullet from this test are presented and discussed. It can be summarise that as the number of layer increases, impact energy also increases. In addition, from the results, it was observed that by using different types of bullets (blunt, hemispherical, conical), there is only a slight difference in values of energy absorbed by the specimen.
\end{abstract}

Keyword: SSGG (Single Stage Gas Gun); GFRP (Glass Fibre Reinforced Polymer); Impact Energy (EI); High Velocity Impact (HVI) 